\title{
Development of Alpha-emitting radioembolization for Hepatocellular Carcinoma: Longitudinal Monitoring of Actinium-225's Daughters Through SPECT Imaging
}

\author{
Yong Du \\ Johns Hopkins University School of Medicine \\ Angel Cortez \\ University of Pittsburgh School of Medicine \\ Mohammadreza Zarisfi \\ Johns Hopkins University School of Medicine \\ Anders Josefsson \\ Johns Hopkins University School of Medicine \\ Rebecca Krimins \\ Johns Hopkins University School of Medicine \\ Eleni Liapi \\ Johns Hopkins University School of Medicine \\ Jessie R Nedrow ( $\nabla$ nedrowj@upmc.edu ) \\ Johns Hopkins School of Medicine https://orcid.org/0000-0002-3465-1296
}

Original research

Keywords: Hepatocellular carcinoma, Lipiodol®, Targeted alpha therapy, Actinium-225, SPECT

Posted Date: December 29th, 2020

DOI: https://doi.org/10.21203/rs.3.rs-134829/v1

License: (c) (i) This work is licensed under a Creative Commons Attribution 4.0 International License.

Read Full License 


\section{Abstract}

Hepatocellular carcinoma is the most common primary liver cancer and the fifth most frequently diagnosed cancer worldwide. Most patients with advanced disease are offered non-surgical palliative treatment options. This work explores the first a-emitting radioembolization for the treatment and monitoring of hepatic tumors. Furthermore, this works demonstrates the first in vivo simultaneous multiple-radionuclide SPECT images of the complex decay chain of an $\left[{ }^{225} \mathrm{Ac}\right] \mathrm{Ac}-\mathrm{labeled}$ agent using a clinical SPECT system to monitor the temporal distribution.

Methods: A DOTA chelator was modified with a lipophilic moiety and radiolabeled with Actinium-225. The resulting agent, $\left[{ }^{225} \mathrm{Ac}\right] \mathrm{Ac}-\mathrm{DOTA}$-TDA, was emulsified in Lipiodol ${ }^{\circledR}$ and evaluated in vivo in mouse model and the VX2 rabbit technical model of liver cancer. SPECT imaging was performed to monitor distribution of the TAT agent and the free daughters.

Results: $\left[{ }^{225} \mathrm{Ac}\right]$ Ac-DOTA-TDA was shown to retain within the HEP2G tumors and VX2 tumor, with minimal uptake within normal tissue. In the mouse model, significant improvements in overall survival were observed. SPECT imaging was able to distinguish between the Actinium-225 agent (Francium-221) and the loss of the longer lived daughter, Bismuth-213.

Conclusion: A TAT agent emulsified in Lipiodol ${ }^{\circledR}$ is capable of targeting liver tumors with minimal accumulation in normal tissue, providing a potential therapeutic agent for the treatment of HCC as well as a variety of hepatic tumors. In addition, SPECT imaging presented here provides a foundation for imaging methodology and protocols that can be rapidly translated into the clinic to monitor Actinium225-labeled agents.

\section{Introduction}

Hepatocellular carcinoma (HCC) is the most common primary liver cancer and the fifth most frequently diagnosed cancer worldwide, as well as the second most frequent cause of cancer death[1-5]. Most patients with advanced disease are offered only non-surgical palliative treatment options, including systemic chemotherapy, radiofrequency ablation, or intra-arterial therapies[6]. Hepatic tumors derive their blood supply primarily from the hepatic artery, whereas normal liver is mainly fed by the portal vein. Image-guided intra-arterial therapies, such as transarterial embolization (TAE), chemoembolization (TACE) and radioembolization (TARE), exploit the arterial route to selectively deliver therapy to tumors. However, these therapies have several limitations, urging the development of new treatment strategies[7, 8].

Ethiodized oil or Lipiodol ${ }^{\circledR}$ is an FDA-approved radio-opaque agent composed of a variety of oils including the ethyl esters of fatty acids of poppy seed oil. Lipiodol® accumulates and remains in liver cancer while clearing out of normal liver tissue when injected via the hepatic artery, providing an excellent vehicle for the selective delivery of therapeutic radionuclides. $\beta$-emitting radiopharmaceutical therapies, such as the commercially available Yttrium-90 glass or resin microspheres have been developed over the 
past 20 years[7, 9] but have shown limited survival benefits in patients with HCC as compared to TACE, the standard of care treatment option. Compared to Yttrium-90 microspheres that accumulate into arterioles, Lipiodol $®$-based $\beta$-emitting agents, such as $\left[{ }^{131} \mathrm{I}\right] \mathrm{l}$-Lipiodol ${ }^{\circledR}$, seem to be directly up taken by tumor cells, resulting in a higher tumor radiation dose[10]. However, HCC patients treated with [ $\left.{ }^{131} 1\right]$ ILipiodol® vs. TACE had similar survival up to three years post-treatment; yet patients that had a portal vein thrombosis or more advanced disease demonstrated a significantly higher mean survival as compared to patients treated with TACE[10]. The incorporation of lodine-131 into Lipiodol囚 is costly and the exploration of alternate $\beta$-emitting radionuclides has been explored, such as chelation of Rhenium188 into Lipiodol $\circledast[11-13]$. Despite initial efficacy of $\left[{ }^{188} \mathrm{Re}\right]$ Re-Lipiodol ${ }^{\circledR}$, the increased hepatic and hematologic toxicities have been a concern[13]. In addition, the low energy transfer of $\beta$-particles introduces the ability of the remaining tumors to develop resistance mechanisms further limiting the treatment options for patients $[14,15]$.

Targeted alpha therapy (TAT) has emerged as a highly potent treatment for cancer[16-18]. The high potency of TAT impacts normal tissue as well, highlighting a need to accurately determine absorbed doses to provide well-defined dosing strategies and effective treatment while minimizing toxicities. Currently, surrogate imaging agents are utilized to evaluate TAT agents; however, complex decay schemes of a-emitting radionuclides are not accurately portrayed using surrogate imaging strategies, urging the development of methodologies and instrumentation to image a-emitting radionuclides. Actinium-225 is one of the a-emitting radionuclides currently being explored in the clinic. Actinium-225 has a complex decay scheme and energy spectrum (Figure 1). Please note, eventhough there are several low abundant $\mathrm{X}$-rays from Actinium-225 and its daughters at the $70 \mathrm{keV}$ to $90 \mathrm{keV}$ energy range, the peak around $80 \mathrm{keV}$ shown in Figure 1 is dominated by the lead X-rays generaged from interactions of high-energy photons with the collimator. This is a well known phenomina in SPECT physics [19]. In addition, this region is also heavily contaminated by downscatter, and thus should not be used for imaging on current clinical SPECT systems. The decay of Actinium-225 and its daughters primarily releases energy as a-particles; however, $\mathrm{Y}$-emissions suitable for SPECT imaging occur, but are in low abundance. The activity administered for TAT is extremely small due to their high potency, compounding the low abundance of rays suitable for imaging and resulting in a small number of y photons for SPECT imaging. Furthermore, the initial alpha decay with its high recoil energy breaks the bonds within the chelate, resulting in the release of the free decay daughters. These daughters may migrate to other organs, complicating dosimetry calculations. Surrogate imaging radionuclides do not replicate the complex decay schemes that are commonly found for a-emitting radionuclides. Therefore, it is urgent and essential to develop quantitative SPECT imaging methodologies specifically for these TAT radionuclides to improve the accuracy and precision of the imaging and the resulting knowledge of the distributions and dose deposition of the agent and its daughters.

In this study we investigate the potential of an $\left[{ }^{225}\right.$ Ac]Ac-labeled TAT Lipiodol ${ }^{\circledR}{ }^{\circledR}$-based agent to selectively target liver tumors for TAT. Furthermore, we evaluated the prospect of using a clinical 
SPECT/CT system to image and monitor the distribution of the $\left[{ }^{225} \mathrm{Ac}\right] \mathrm{Ac}$-labeled TAT Lipiodol ${ }^{\circledR}$-based agent as well as its daughters in the VX2-tumor bearing rabbit model over a 6-day window.

\section{Materials And Methods}

\section{Reagents}

All chemicals were purchased from Sigma-Aldrich Chemical Co. (ST. Louis, MO, USA) or Thermo Fisher Scientific (Pittsburgh, PA, USA), unless otherwise specified. DOTA-tris(tert-butyl ester) was purchased from Macrocyclics, Inc. (Dallas, TX, USA). Indium-111 ([ $\left[^{111} \mathrm{In}\right] \mathrm{InCl} 3$ ) was purchased from BWZXT ITG Canada, Inc. (Ottawa, ON, Canada). Actinium-225 nitrate was purchased from Oak Ridge National Laboratory (Oak Ridge, TN, USA).

Chemical Syntheses. DOTA-tris(t-Bu ester)-TDA was synthesized as previously described with modifications [20]. Briefly, the DOTA-tris(t-Bu) ester (50 mg, $0.09 \mathrm{mmol})$ and HBTU $(0.168 \mathrm{mg}, 0.44 \mathrm{mmol})$ were dissolved in $2 \mathrm{~mL}$ of DMF. The resulting solution was stirred for 30 minutes at room temperature (RT) then 1-tetradecylamine (TDA) $(22 \mathrm{mg}, 0.11 \mathrm{mmol})$ and DIPEA $(76 \mu \mathrm{l}, 0.44 \mathrm{mmol})$ were added to the solution, which was stirred overnight at RT. The solution was extracted with ethyl acetate washed with water $(2 \mathrm{x})$ then brine $(1 \mathrm{x})$. The ethyl acetate solution was dried over $\mathrm{MgSO}_{4}$, filtered and the ethyl acetate was evaporated under vacuum to yield a yellow powder. The DOTA-tris(t-Bu ester)-TDA was purified by silica gel chromatography (eluent: hexane/ethyl acetate, $3 / 1 \mathrm{v} / \mathrm{v}$ ), purity confirmed by TLC and the product was used without further confirmation.

DOTA-TDA. DOTA-tris(t-Bu ester)-TDA ( $30 \mathrm{mg}, 0.04 \mathrm{mmol}$ ) was dissolved in trifluoroacetic acid (125 $\mu \mathrm{l})$ and dichloromethane $(400 \mu \mathrm{l})$ and stirred overnight at RT. The solvent was evaporated under vacuum yield a pure white solid, $51.3 \%$ was recovered. DOTA-TDA was successfully synthesized (overall yield $53.1 \%$ ) and confirmed by mass spectrometry. DOTA-TDA was confirmed by using a Thermo Scientific Q Exative Plus Orbitrap Mass Spectrometer coupled with a Vanquish UPLC system at the Metabolomics Facility at Johns Hopkins School of Medicine (Director - Dr. Anne Le) $(M+H)$ : calculated 600.4336, found 600.4316; (M-H) calculated 598.4185 found 598.4203.

Radiolabeling of DOTA-TDA. For radiolabeling the following were added to acid wash Eppendorf tube $1 \mu \mathrm{l}$ of Indium-111, $40 \mu \mathrm{l}$ of $0.4 \mathrm{M} \mathrm{NaOAc}$ buffer $\mathrm{pH}=4.5$, and $1 \mu \mathrm{l}$ of DOTA-TDA $(1 \mu \mathrm{g} / \mu \mathrm{l})$. The solution was heated at $95^{\circ} \mathrm{C}$ for 15 minutes and purified using a Waters Sep-Pak C18 (Milford, MA, USA). [ $\left.{ }^{111} \mathrm{In}\right] \mathrm{In}$ DOTA-TDA was eluted with ethanol, dried, and resuspended in sterile PBS with greater that $95 \%$ radiopurity as determined by thin layer chromatography (TLC).

For Actinium-225, $5 \mu$ l of Actinium-225 solution, $5 \mu \mathrm{l}$ of $2 \mathrm{M}$ Tris buffer $\mathrm{pH}=7$, and $2.5 \mu \mathrm{L}$ of DOTA-TDA were added to an acid washed tube, heated at $95^{\circ} \mathrm{C}$ for 15 minutes. Radiolabeling yields were $\geq 95 \%$; $\left[{ }^{225} \mathrm{Ac}\right]$ Ac-DOTA-TDA was used without further purification. 
Partition Coefficient (Log P). Log P were determined for the radiolabeled conjugates, $\left[{ }^{111} \mathrm{In}\right] \mathrm{In}$ - or $\left[{ }^{225}\right.$ Ac]Ac-DOTA-TDA, were dissolved in $1 \mathrm{~mL}$ of octanol in a centrifuge tube. The centrifuge tube was vigorously vortexed for a minute with $1 \mathrm{~mL}$ of $0.9 \%$ saline. The tube was centrifuged at $1000 \mathrm{rpm}$ for 5 minutes and allowed to sit for 30 minutes until the phases were clearly separated. The organic phase (Octanol) and the aqueous phase (Saline) were collected separately and counted at equilibrium of Actinium-225 in an automatic $y$-well counter (Perkin-Elmer 2470 WIZARD ${ }^{2}$ ) Automatic Gamma Counter, Waltham, MA, USA). The Log P values were calculated using the following formula: $\log P=\log$ ([Counts $\left.]_{\text {octanol }} /[\text { Counts }]_{\text {saline }}\right)$.

\section{Animal Studies}

Animal studies were performed using 7-8-week-old healthy male NCG mice obtained from Charles River Laboratories (Wilmington, MA, USA). Adult New Zealand rabbits were used weighing approximately $4 \mathrm{~kg}$ (Myrtle's Rabbitry, Thompson Station, TN, USA). All animal studies were approved by the Animal Care and Use Committee of the Johns Hopkins University School of Medicine. For tumor retention and survival studies the mice were injected subcutaneously in the right flank with approximately $1 \times 10^{6} \mathrm{HEP} 2 \mathrm{G}$ cells in $100 \mu \mathrm{L}$ of sterile PBS and Matrigel (1:1).

\section{Mouse Model}

Tumor Retention. Biodistribution studies were carried out as previously described in healthy NCG male mice ( $n=3$ /group) bearing HEP2G subcutaneous tumors [21, 22]. To assess tumor retention the mice were divided into two groups that were injected intratumorally with ${ }^{111}$ In-DOTA-TDA $(37 \mathrm{kBq}, 60 \mu \mathrm{L})$ alone or

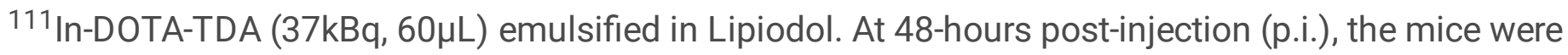
euthanized. The blood, heart, lungs, liver, spleen, kidneys, stomach (with content), intestines (with content), bone, muscle, and tumors were harvested, weighed, and measured in an automatic $\gamma$-well counter. The percentage of injected dose per gram (\%ID/g) was calculated by comparison with a weighed, diluted standard.

Therapeutic Efficacy: Survival Studies. Subcutaneous liver cancer models were used to evaluate the therapeutic efficacy of $\left[{ }^{225}\right.$ Ac]Ac-DOTA-TDA/Lipiodol emulsions. The following treatments were administered in subcutaneously HEP2G tumors $\left(266.5 \pm 23.2 \mathrm{~mm}^{3}\right)$ : 1 . Saline $(60 \mu \mathrm{L}) 2$. Lipiodol alone $(60 \mu \mathrm{L})$ or 3. $\left[{ }^{225} \mathrm{Ac}\right]$ Ac-DOTA-TDA/Lipiodol $(37 \mathrm{kBq}, 60 \mu \mathrm{L})$. Mice were observed for signs of pain and distress (lethargy, hunched back, paralysis, etc.), weight loss, and tumor size [volume $=0.5$ (length $x$ width $\left.^{2}\right)$ ]; mice were euthanized if one of the following conditions were met: 1 . weight loss $(>20 \%) ; 2$. primary tumor reaching $1,500 \mathrm{~mm}^{3} ; 3$. signs of pain or distress; or 4.180 days post-treatment. Survival fractions were plotted as a Kaplan-Meier survival curve using Prism 8 (GraphPad; La Jolla, CA, USA).

\section{VX2 Rabbit Model}


Anesthesia. Rabbits were administered a mixture of ketamine $(30-50 \mathrm{mg} / \mathrm{kg})$ and xylazine $(3-5 \mathrm{mg} / \mathrm{kg})$ intramuscularly prior to tumor harvesting, implantation, and imaging. Anesthesia was maintained with $3 \%$ isoflurane in oxygen as well as additional injections of the ketamine/xylazine solution.

Ultrasound Guided Tumor Implantation. The implantation of the VX2 tumor was done as previously described with modifications[23]. Briefly, the solid VX2 tumor for implantation was obtained from a carrier rabbit that had been injected intramuscularly in the thigh approximately 2 weeks prior. The VX2 tumor was harvested, placed in $0.9 \%$ sodium chloride, and minced into small pieces. The minced tumor was prepared in a 16-gauge Angiocath ( 2 inches long), using ultrasound guidance, the needle punctured the liver and the tumor pieces were pushed into the liver with a guide wire. Manual compression was administered after the needle was removed.

Intrahepatic Arterial Injection including Agents and Dosing. Hepatic angiography was performed as previously described[23]. Briefly, rabbits with VX2 hepatic tumors, confirmed by ultrasound were anesthetized and surgical cutdowns were performed to expose the femoral artery. A 4-F sheath (Cook) was inserted to allow a 2-F catheter tip (JB1 catheter; Cook) to access the common hepatic artery. Fluoroscopy was utilized to select the arterial branch and radioembolization was performed.

SPECT Imaging in VX2-bearing Rabbit. SPECT/CT imaging was performed on a Siemens Symbia T16 SPECT/CT system. The anesthetized rabbit $(n=1)$ was placed supine on the imaging bed with the whole body in the field-of-view. The images were acquired at 24 hours, 72 hours and 6 days p.i.. Projection data were acquired in 120 views over $360^{\circ}$ with a $128 \times 128$ matrix and $4.795 \mathrm{~mm}$ pixel size. The imaging time were 75 seconds per view for the 24-hour imaging, and 90 seconds per view for the 72 -hour and 6-day imaging. The high-energy collimators were used. Data were binned into two energy windows - $20 \%$ centered at $440.45 \mathrm{keV}$ peak of Bismuth-213 and $20 \%$ centered at $218 \mathrm{keV}$ peak of Francium-221, respectively. Both isotopes are daughters of Actinium-225 decay and emit gamma photons that can be imaged by SPECT. Francium-221 has a short half-life of 4.9 minutes, therefore its distribution represents those of Actiniun-225's. Bismuth-213 has a half-life of approximately 45.6 minutes and could have redistributed from the parent isotope. After SPECT acquisition, a CT scan was performed to provide anatomical images and an attenuation map. The SPECT images were reconstructed with Siemens Flash3D OSEM algorithm with compensation for attenuation and resolution[24]. A total of 6 iterations with 15 subsets per iteration were used.

Ex Vivo Biodistribution VX2-bearing and non-tumor bearing Rabbits. Biodistribution studies were carried out following the SPECT imaging approximately 6-days post-injection of $\left[{ }^{225}\right.$ Ac]Ac-DOTA-TDA (0.49 MBq) emulsified in $1 \mathrm{~mL}$ of Lipiodol in the VX2-bearing rabbit $(\mathrm{n}=1)$ and at approximately 24 hours for nontumor bearing rabbits $(n=2)$. At the end timepoint the rabbits were euthanized (Ethansol $\circledast, 1 \mathrm{~mL}$ minimum then $1 \mathrm{~mL} / 4.5 \mathrm{~kg}$ ). The blood, heart, lungs, liver, spleen, kidneys, stomach, intestines, marrow, femur+marrow, muscle, gall bladder, bile, and if applicable the tumor were harvested, weighted, and measure in an automatic $\gamma$-well counter and/or a Capintec CRC-7 dose calibrator. The percentage of 
injected dose per gram (\%ID/g) was calculated based on the dose injected and converted to $\mathrm{Bq} / \mathrm{g}$ using an efficiency coefficient.

Stastical Analysis was performed using the software Graphpad Prism 8. All data are presented as mean \pm SD. Tumor rententions were compared using 2-way ANOVA. Survival studies used Kaplan-Meier curves that were analyzed using the Log Rank (Mantel-Cox) test. Values were considered significant when $P<0.05$.

\section{Results}

Radiochemistry. [ ${ }^{111}$ In] In-DOTA-TDA was radiolabeled at a specific activity of $2.13 \mathrm{MBq} / \mathrm{nmol}$ with greater than $95 \%$ radiopurity following purification. The $\left[{ }^{225} \mathrm{Ac}\right]$ Ac-DOTA-TDA was radiolabeled at a specific activity of $0.36 \mathrm{MBq} / \mathrm{nmol}$ with greater than $95 \%$ radiolabeled yield.

Determining Partition Coefficients (LogP). The LogP for [ $\left.{ }^{111} \mathrm{In}\right] \operatorname{In}$-DOTA-TDA was $1.57 \pm 0.01$ and the LogP for $\left[{ }^{225}\right.$ Ac]Ac-DOTA-TDA was $1.65 \pm 0.02$. For comparison, doxorubicin, a chemotherapeutic used for TACE, has a reported LogP of 1.27[25].

Tumor Retention of ${ }^{111}$ In-DOTA-TDA-LipiodoI®. The retention of $\left[{ }^{111} \mathrm{In}\right] \mathrm{In}$-DOTA-TDA alone or as a Lipiodol ${ }^{\circledR}$ emulsion (Figure 2) were compared 48-hours p.i. using a 2-way ANOVA test in HEP2G tumorbearing mice. The $\left[{ }^{111}\right.$ In] In-DOTA-TDA-Lipiodol® emulsion had significantly higher uptake within the tumor as compared to [ $\left.{ }^{111} \mathrm{In}\right] \mathrm{In}$-DOTA-TDA alone after 48 hours $(216 \pm 145 \% \mathrm{ID} / \mathrm{g}$ vs. $20.0 \pm 15.9 \% \mathrm{ID} / \mathrm{g}$; $p \leq 0.0001$ ), supporting the statement that Lipiodol ${ }^{\circledR}{ }^{\circledR}$ is able to retain the proposed agents in a model of liver cancer.

Therapeutic Efficacy: Survival Studies. Kaplan-Meier survival curves (Figure 3) showed significant improvement in survival of HEP2G tumor-bearing mice when treated with $\left[{ }^{225}\right.$ Ac]Ac-DOTA-TDA-Lipiodol ${ }^{\circledR}$ as compared to both the untreated control $(p<0.005)$ and Lipiodol ${ }^{\circledR}$ alone $(p<0.001)$. The median survival for $\left[{ }^{225} \mathrm{Ac}\right]$ Ac-DOTA-TDA-Lipiodol ${ }^{\circledR}$ treated mice was 45 days, ranging from 26-87 days, as compared to 24-days (untreated, [15-29 days]) and 26-days (Lipiodol $\circledast$ alone, [15-40 days]). In addition, the rate of tumor growth in the $\left[{ }^{225} \mathrm{Ac}\right]$ Ac-DOTA-TDA-Lipiodol $\circledast$ treated group was slowed as demonstrated by the individual tumor growth curves (Figure 4).

SPECT Imaging in VX2-bearing Rabbit. The fused SPECT/CT images from 24, 72, and 144-hours p.i. shown in Figure 5 demonstrated that the activity of $\left[{ }^{225}\right.$ Ac]Ac-DOTA-TDA-Lipiodol ${ }^{\circledR}$ agent accumulated mainly in the VX2 tumor with gradual clearance out of normal liver tissue. At 72 hours and 6-day p.i., the $\left[{ }^{225} \mathrm{Ac}\right]$ Ac-DOTA-TDA-Lipiodol ${ }^{\circledR}$ agent is concentrated within the tumor; however, the figures clearly show that there were differences in the distributions between the Francium-221 energy window and Bismuth213 energy window - there was increased signal in the normal liver tissue above the tumor in the Bismuth213 window, most likely associated with the escape of the free Bismuth-213 daughter from TAT agent. 
Ex-Vivo Biodistribution in VX2-bearing Rabbit. Following the SPECT imaging, biodistribution studies were carried out in the VX2-tumor bearing rabbit at 6-days p.i. of the [ $\left.{ }^{225} \mathrm{Ac}\right] \mathrm{Ac}$-DOTA-TDA-Lipiodol ${ }^{\circledR}$ emulsion (Table 1). Accumulation of the TAT agent was predominately in the VX2 tumor $(40.6 \% \mathrm{ID} / \mathrm{g})$. The next highest uptake was seen in the normal liver tissue $(0.23 \% \mathrm{ID} / \mathrm{g})$, resulting in having approximately 177:1 tumor to liver ratio. The TAT agent accumulation in normal tissues were minimal, only slightly above background as determined by uptake in the muscle $(0.001 \% \mathrm{ID} / \mathrm{g})$. This low uptake in normal tissue is consistent with the ex-vivo biodistributions in non-tumor bearing rabbits $(n=2)$ at approximately 24-hours p.i. via the hepatic artery as shown in Table 1.

\section{Discussion}

Due to its indolent course, most patients with HCC have advanced and unresectable disease at the time of diagnosis and are being offered only non-surgical palliative treatment options[6, 7]. In addition, the liver is also the most common site for metastatic disease, including colorectal liver metastases. Intraarterial therapies are widely used for treatment of patients with HCC or metastatic liver cancer[6]. Nevertheless, these therapies have shown limited survival benefit for patients with HCC, urging the need for developing novel improved therapeutic strategies [6, 26-30].

$\beta$-emitting radiopharmaceutical agents have shown promising results but the survival benefits in patients with HCC are comparable to the standard of care, TACE[31]. TAT provides highly potent agents[32-45] that are highly damaging to tumor cells, effectively causing irreparable DNA damage to tumor cells, needing only a few a-tracks as compared to $10^{3}-10^{4}$ tracks for a $\beta$-emitting agents. Consequently, there is growing interest in TAT agents for cancer therapy[46-48]. Recently, the first in human experience with [ $\left.{ }^{213} \mathrm{Bi}\right] \mathrm{Bi}$ DOTATOC, a TAT agent for neuroendocrine cancer, was selectively targeted via the hepatic artery to treat metastatic disease in the liver of neuroendocrine cancer patients not responding to standard therapies as well as [ ${ }^{177}$ Lu]Lu-DOTATOC, a $\beta$-emitting targeted therapy. Kratochwil, et. al. demonstrated in a small subset of patients that [ $\left.{ }^{213} \mathrm{Bi}\right] \mathrm{Bi}$-DOTATOC was able to overcome refractory disease and provided positive responses in patients[49]. Biomarkers for targeted radiopharmaceutical therapies are limited for HCC; however, the selective deliver of therapies via the hepatic artery for primary liver cancers are common. In this study, we investigated the potential to exploit intra-arterial delivery of the TAT agent, $\left[{ }^{225}\right.$ Ac]Ac-DOTATDA-Lipiodol ${ }^{\circledR}$, for its ability to target primary liver tumors as well as its therapeutic efficacy in a mouse model of HCC.

Lipiodol $\circledast$ has been demonstrated to have high selectivity and retention in the liver [50]; Lipiodol $\circledast$ has shown to be retained in hepatic tumors for months [51]. [ ${ }^{188} \mathrm{Re}$ Re-labeled chelators, modified to introduce lipophilicity, have been successfully incorporated into Lipiodol ${ }^{\circledR}$ for $\beta$-emitting radiopharmaceutical therapy[52]. Here, we used a similar approach and altered the DOTA chelator, which is suitable for $\left[{ }^{225} \mathrm{Ac}\right] \mathrm{Ac}-l$ labeling, by incorporating an alkyl side chain to introduce lipophilicity. The resulting agent, DOTA-TDA, was radiolabeled with both Indium-111 and Actnium-225 in high radiolabeling yields and purities. Both the $\left[{ }^{111} \mathrm{In}\right] \mathrm{In}$ - and $\left[{ }^{225} \mathrm{Ac}\right]$ Ac-DOTA-TDA were shown to be lipophilic as determined by the 
partition coefficients, helping them to be emulsified into Lipiodol®. The emulsification of $\left[{ }^{111}\right.$ In] In-DOTATDA with Lipiodol ${ }^{\circledR}$ resulted in an approximate 11 -fold increase in retention of the radiolabel agent within the mouse model of primary liver cancer. The potency of the TAT agent, $\left[{ }^{225}\right.$ Ac]Ac-DOTA-TDA-Lipiodol ${ }^{8}$, was evaluated and demonstrated significant increases in survival in TAT treated mice as compared to mice receiving no treatment or Lipiodol $\circledast$ alone. $\left[{ }^{225}\right.$ Ac]Ac-DOTA-TDA-Lipiodol ${ }^{\circledR}$ treated mice almost doubled (1.8x) their median survival as compared to the control groups, with the first TAT-treated mouse not reaching its endpoint until the median survival of the control. The preliminary results in the mouse model of primary liver cancer are promising, and we further evaluated the TAT agent in a technical model to confirm selective delivery and retention via intra-arterial delivery.

The rabbit VX2 model provides a technical animal model for intra-arterial injections, allowing us to explore the selective delivery of $\left[{ }^{225}\right.$ Ac]Ac-DOTA-TDA-Lipiodol ${ }^{\circledR}$ to a hepatic tumor. We have confirmed delivery, accumulation, and retention of the TAT-Lipiodol® agents within the VX2 tumor by ex-vivo biodistribution, and in-vivo SPECT imaging of Acintium-225's daughters (Francium-221 and Bismuth-213) using a clinical SPECT/CT system. Even though feasibility of SPECT imaging of Actinium-225 decay chain has been demonstrated $[53,54]$-- to our knowledge, this work is the first to demonstrate it can be done longitudinally in-vivo on a clinical SPECT/CT system with low activities, allowing for dosimetry without the use of a surrogate imaging agent. Furthermore, the fact that the two energy windows showed different distributions indicates the importance of using SPECT to monitor both parent and daughter isotopes. Clearance from normal tissues including the liver was seen in early biodistribution studies in non-tumor-bearing rabbits at approximately 24 hours p.i. of $\left[{ }^{225}\right.$ Ac]Ac-DOTA-TDA-Lipiodol ${ }^{\circledR}$ with the highest uptake, less than $0.2 \% \mathrm{ID} / \mathrm{g}$, in the normal liver. The VX2-bearing rabbit's biodistribution showed that the normal liver had slightly higher uptake $(0.23 \% \mathrm{ID} / \mathrm{g})$ of $\left[{ }^{225} \mathrm{Ac}\right]$ Ac-DOTA-TDA-Lipiodol ${ }^{8}$ at 6-days p.i., most likely associated with clearance of the TAT agent from the VX2 tumor, which had $40.6 \% \mathrm{ID} / \mathrm{g}$ uptake at 6-days p.i.. This is further confirmed by the SPECT images that demonstrated the clearance of the TAT agent from the normal liver tissue over time. More importantly, the SPECT images also demonstrated the differences in the distribution of Actinium-225/Francium-221 from that of free Bismuth-213, indicating the importance of obtaining the daughter isotopes' distribution for accurate dosimetry for TAT.

\section{Conclusion}

In summary, these studies demonstrate that the developed TAT agent, $\left[{ }^{225}\right.$ Ac]Ac-DOTA-TDA-Lipiodol ${ }^{\circledR}$, has promise as a therapeutic agent for hepatic tumors, including primary liver tumors. This work highlights that the combination of a TAT agent labeled with a long-lived a-emitting radionuclide emulsified with Lipiodol ${ }^{\circledR}$ is capable of being selectively delivered to hepatic tumors, delivering a highly potent therapeutic dose to hepatic tumors over an extended period of time. Furthermore, we have shown that an $\left[{ }^{225}\right.$ Ac]Ac-labeled TAT agent can be imaged and longitudinally monitored through clinical SPECT imaging using its daughters Francium-221 and Bismuth-213 even with very low injected activity. The development of SPECT imaging methodologies to monitor the complex decay scheme of a-emitting 
radionuclides, and their daughters, such as Actinium-225, are important for accurately monitoring the distribution of TAT agents in-vivo over time through molecular imaging, providing essential

pharmacokinetic data to optimize the therapeutic efficacy of $\left[{ }^{225}\right.$ Ac]Ac-DOTA-TDA-Lipiodol ${ }^{\circledR}$. More importantly, these SPECT imaging methodologies can be implemented for [ $\left.{ }^{225} \mathrm{Ac}\right] \mathrm{Ac}$-labeled TAT agents currently in clinical trials helping to develop well-defined dosing strategies and optimal patient-specific treatment planning.

\section{Declarations}

Funding: NIH R01CA239041, Johns Hopkins Department of Radiology's Walter \& Mary Ciceric Research Award and the Frank P. \& Elizabeth K. Leo Research Award.

Conflicts of interest: Drs. Nedrow and Liapi currently hold a patent application on the presented work (PCT/US2019/029051).

Ethics Approval: All animal studies were approved by the Animal Care and Use Committee of the Johns Hopkins University School of Medicine.

Consent to participate: N/A

Consent to publication: N/A

Code Availability: N/A

\section{ACKNOWLEDGEMENTS}

The Actinium-225 used in this research was supplied by the Isotope Program within the Office of Nuclear Physics in the Department of Energy's Office of Science. This work is supported by the NIH R01CA239041 as well as the Johns Hopkins Department of Radiology's Walter \& Mary Ciceric Research Award and the Frank P. \& Elizabeth K. Leo Research Award.

\section{References}

1. Jemal A, Bray F, Center MM, Ferlay J, Ward E, Forman D. Global cancer statistics. Cancer J Clin. 2011;61:69-90. doi:10.3322/caac.20107.

2. Center MM, Jemal A. International trends in liver cancer incidence rates. Cancer epidemiology, biomarkers \& prevention: a publication of the American Association for Cancer Research. cosponsored by the American Society of Preventive Oncology. 2011;20:2362-8. doi:10.1158/10559965.EPI-11-0643.

3. De P, Dryer D, Otterstatter MC, Semenciw R. Canadian trends in liver cancer: a brief clinical and epidemiologic overview. Curr Oncol. 2013;20:e40-3. doi:10.3747/co.20.1190. 
4. Simard EP, Ward EM, Siegel R, Jemal A. Cancers with increasing incidence trends in the United States: 1999 through 2008. CA: a cancer journal for clinicians. 2012. doi:10.3322/caac.20141.

5. Liapi E, Geschwind JFH. Transcatheter and ablative therapeutic approaches for solid malignancies. J Clin Oncol. 2007;25:978-86. doi:Doi 10.1200/Jco.2006.09.8657.

6. Liapi E, Geschwind JF. Transcatheter and ablative therapeutic approaches for solid malignancies. J Clin Oncol. 2007;25:978-86. doi:10.1200/JC0.2006.09.8657.

7. Lee KH, Liapi EA, Cornell C, Reb P, Buijs M, Vossen JA, et al. Doxorubicin-loaded QuadraSphere microspheres: plasma pharmacokinetics and intratumoral drug concentration in an animal model of liver cancer. Cardiovasc Intervent Radiol. 2010;33:576-82. doi:10.1007/s00270-010-9794-1.

8. Liapi E, Georgiades CC, Hong K, Geschwind JF. Transcatheter arterial chemoembolization: current technique and future promise. Tech Vasc Interv Radiol. 2007;10:2-11. doi:S1089-2516(07)00070-4 [pii].

9. 1053/j.tvir.2007.08.008.

10. Lewandowski RJ, Geschwind JF, Liapi E, Salem R. Transcatheter intraarterial therapies: rationale and overview. Radiology. 2011;259:641-57. doi:10.1148/radiol.11081489.

11. Marelli L, Shusang V, Buscombe JR, Cholongitas E, Stigliano R, Davies N, et al. Transarterial injection of (131) I-lipiodol, compared with chemoembolization, in the treatment of unresectable hepatocellular cancer. J Nucl Med. 2009;50:871-7. doi:10.2967/jnumed.108.060558.

12. Bernal P, Raoul JL, Vidmar G, Sereegotov E, Sundram FX, Kumar A, et al. Intra-arterial rhenium-188 lipiodol in the treatment of inoperable hepatocellular carcinoma: results of an IAEA-sponsored multination study. Int J Radiat Oncol Biol Phys. 2007;69:1448-55. doi:10.1016/j.jijrobp.2007.05.009.

13. Zanzonico PB, Divgi C. Patient-specific radiation dosimetry for radionuclide therapy of liver tumors with intrahepatic artery rhenium-188 lipiodol. Semin Nucl Med. 2008;38:30-9. doi:10.1053/j.semnuclmed.2007.10.005.

14. Bernal P, Raoul JL, Stare J, Sereegotov E, Sundram FX, Kumar A, et al. International Atomic Energy Agency-sponsored multination study of intra-arterial rhenium-188-labeled lipiodol in the treatment of inoperable hepatocellular carcinoma: results with special emphasis on prognostic value of dosimetric study. Semin Nucl Med. 2008;38:40-5. doi:10.1053/j.semnuclmed.2007.10.006.

15. Sofou S. Radionuclide carriers for targeting of cancer. Int J Nanomedicine. 2008;3:181-99.

16. McDevitt MR, Sgouros G, Sofou S. Targeted and Nontargeted alpha-Particle Therapies. Annu Rev Biomed Eng. 2018;20:73-93. doi:10.1146/annurev-bioeng-062117-120931.

17. [Cancer of the prostate. Xofigo in the hospitals]. Perspect Infirm. 2015;12:61.

18. Radium - 223 (Xofigo) for prostate cancer. Med Lett Drugs Ther. 2013;55:79-80.

19. Kratochwil C, Bruchertseifer F, Rathke H, Bronzel M, Apostolidis C, Weichert W, et al. Targeted alphaTherapy of Metastatic Castration-Resistant Prostate Cancer with 225Ac-PSMA-617: Dosimetry Estimate and Empiric Dose Finding. J Nucl Med. 2017;58:1624-31.

doi:10.2967/jnumed.117.191395.

Page $11 / 19$ 
20. de Jong HW, Beekman FJ, Viergever MA, van Rijk PP. Simultaneous (99 m)Tc/(201)TI dual-isotope SPET with Monte Carlo-based down-scatter correction. Eur J Nucl Med Mol Imaging. 2002;29:106371. doi:10.1007/s00259-002-0834-1.

21. Grogna M, Cloots R, Luxen A, Jérôme C, Desreux J-F, Detrembleur C. Design and synthesis of novel DOTA(Gd3+)-polymer conjugates as potential MRI contrast agents. J Mater Chem. 2011;21:1291726. doi:10.1039/C1JM00005E.

22. Josefsson A, Nedrow JR, Park S, Banerjee SR, Rittenbach A, Jammes F, et al. Imaging, Biodistribution, and Dosimetry of Radionuclide-Labeled PD-L1 Antibody in an Immunocompetent Mouse Model of Breast Cancer. Cancer Res. 2016;76:472-9. doi:10.1158/0008-5472.CAN-15-2141.

23. Nedrow JR, Josefsson A, Park S, Back T, Hobbs RF, Brayton C, et al. Pharmacokinetics, microscale distribution, and dosimetry of alpha-emitter-labeled anti-PD-L1 antibodies in an immune competent transgenic breast cancer model. EJNMMI Res. 2017;7:57. doi:10.1186/s13550-017-0303-2.

24. Lee KH, Liapi E, Buijs M, Vossen J, Hong K, Georgiades C, et al. Considerations for implantation site of VX2 carcinoma into rabbit liver. J Vasc Interv Radiol. 2009;20:113-7. doi:10.1016/j.jvir.2008.09.033.

25. Vija AH, Hawman EG, Engdahl JC. Analysis of a SPECT OSEM reconstruction method with 3D beam modeling and optional attenuation correction: phantom studies. 2003 IEEE Nuclear Science Symposium Conference Record (IEEE Cat No03CH37515); 2003. p. 2662-6 Vol.4.

26. Martin YC. Exploring QSAR: Hydrophobic, Electronic, and Steric Constants C, Hansch A, Leo, Hoekman D. American Chemical Society, Washington DC 1995. Xix + 348 pp. $22 \times 28.5 \mathrm{~cm}$. Exploring QSAR: Fundamentals and Applications in Chemistry and Biology. C. Hansch, Leo A. American Chemical Society, Washington, DC. 1995. Xvii + 557 pp. 18.5 × 26 cm. ISBN 0-8412-2993-7 (set). $\$ 99.95$ (set). Journal of Medicinal Chemistry. 1996;39:1189-90. doi: 10.1021/jm950902o .

27. Liapi E, Geschwind JF. Combination of local transcatheter arterial chemoembolization and systemic anti-angiogenic therapy for unresectable hepatocellular carcinoma. Liver Cancer. 2012;1:201-15. doi:10.1159/000343835.

28. Liapi E, Geschwind JF, Vali M, Khwaja AA, Prieto-Ventura V, Buijs M, et al. Assessment of tumoricidal efficacy and response to treatment with 18F-FDG PET/CT after intraarterial infusion with the antiglycolytic agent 3-bromopyruvate in the VX2 model of liver tumor. J Nucl Med. 2011;52:225-30. doi:10.2967/jnumed.110.083162.

29. Liapi E, Geschwind JF. Interventional oncology: new options for interstitial treatments and intravascular approaches: targeting tumor metabolism via a loco-regional approach: a new therapy against liver cancer. J Hepatobiliary Pancreat Sci. 2010;17:405-6. doi:10.1007/s00534-009-0236-x.

30. Liapi E, Geschwind JF. Chemoembolization for primary and metastatic liver cancer. Cancer J. 2010;16:156-62. doi:10.1097/PP0.0b013e3181d7e905.

31. Liapi E, Geschwind JF. Intra-arterial therapies for hepatocellular carcinoma: where do we stand? Ann Surg Oncol. 2010;17:1234-46. doi:10.1245/s10434-010-0977-4. 
32. Kim DY, Han KH. Transarterial chemoembolization versus transarterial radioembolization in hepatocellular carcinoma: optimization of selecting treatment modality. Hepatol Int. 2016;10:88392. doi:10.1007/s12072-016-9722-9.

33. Ballangrud AM, Yang WH, Charlton DE, McDevitt MR, Hamacher KA, Panageas KS, et al. Response of LNCaP spheroids after treatment with an alpha-particle emitter (213Bi)-labeled anti-prostate-specific membrane antigen antibody (J591). Cancer Res. 2001;61:2008-14.

34. Ballangrud AM, Yang WH, Hamacher KA, McDevitt MR, Ma D, Scheinberg DA, et al. Relative efficacy of the alpha-particle emitters Bi-213 and Ac-225 for radioimmunotherapy against micrometastases. Proc AACR. 2000;41:289.

35. Bloomer WD, McLaughlin WH, Adelstein SJ, Wolf AP. Therapeutic applications of Auger and alpha emitting radionuclides. Strahlentherapie. 1984;160:755-7.

36. Bloomer WD, McLaughlin WH, Neirinckx RD, Adelstein SJ, Gordon PR, Ruth TJ, et al. Astatine-211tellurium radiocolloid cures experimental malignant ascites. Science. 1981;212:340-1.

37. Humm JL, Chin LM. A model of cell inactivation by alpha-particle internal emitters. Radiat Res. 1993;134:143-50.

38. Kassis Al, Harris CR, Adelstein SJ, Ruth TJ, Lambrecht R, Wolf AP. The Invitro Radiobiology of Astatine-211 Decay. Radiat Res. 1986;105:27-36.

39. Kozak RW, Atcher RW, Gansow OA, Friedman AM, Hines JJ, Waldmann TA. Bismuth-212-Labeled Anti-Tac Monoclonal-Antibody - Alpha-Particle-Emitting Radionuclides As Modalities for Radioimmunotherapy. Proc Natl Acad Sci USA. 1986;83:474-8.

40. Kurtzman SH, Russo A, Mitchell JB, DeGraff W, Sindelar WF, Brechbiel MW, et al. Bismuth-212 Linked to An Antipancreatic Carcinoma Antibody - Model for Alpha-Particle-Emitter Radioimmunotherapy. J Natl Cancer Inst. 1988;80:449-52.

41. Macklis RM, Kinsey BM, Kassis Al, Ferrara JLM, Atcher RW, Hines JJ, et al. Radioimmunotherapy with Alpha-Particle Emitting Immunoconjugates. Science. 1988;240:1024-6.

42. McDevitt MR, Ma D, Lai LT, Simon J, Borchardt P, Frank RK, et al. Tumor therapy with targeted atomic nanogenerators. Science. 2001;294:1537-40.

43. Raju MR, Eisen Y, Carpenter S, Inkret WC. Radiobiology of Alpha-Particles.3. Cell Inactivation by Alpha-Particle Traversals of the Cell-Nucleus. Radiat Res. 1991;128:204-9.

44. Ballangrud AM, Yang WH, Palm S, Enmon R, Borchardt PE, Pellegrini VA, et al. Alpha-Particle Emitting Atomic Generator (Actinium-225)-Labeled Trastuzumab (Herceptin) Targeting of Breast Cancer Spheroids: Efficacy versus HER2/neu Expression. Clin Cancer Res. 2004;10:4489-97.

45. Imam SK. Advancements in cancer therapy with alpha-emitters: a review. IntJ RadiatOncol BiolPhys. 2001;51:271-8.

46. McDevitt MR, Sgouros G, Finn RD, Humm JL, Jurcic JG, Larson SM, et al. Radioimmunotherapy with alpha-emitting nuclides. Eur J Nucl Med. 1998;25:1341-51. 
47. Rosenblat TL, McDevitt MR, Mulford DA, Pandit-Taskar N, Divgi CR, Panageas KS, et al. Sequential cytarabine and alpha-particle immunotherapy with bismuth-213-lintuzumab (HuM195) for acute myeloid leukemia. Clin Cancer Res. 2010;16:5303-11. doi:1078-0432.CCR-10-0382 [pii].

48. $1158 / 1078$ - 0432.CCR-10-0382.

49. Sgouros G. Alpha-particles for targeted therapy. Adv Drug Deliv Rev. 2008;60:1402-6. doi:S0169409X(08)00121-X [pii].

50. 1016/j.addr.2008.04.007.

51. Milenic DE, Garmestani K, Brady ED, Albert PS, Abdulla A, Flynn J, et al. Potentiation of high-LET radiation by gemcitabine: targeting HER2 with trastuzumab to treat disseminated peritoneal disease. Clin Cancer Res. 2007;13:1926-35. doi:13/6/1926 [pii].

52. $1158 / 1078$ - 0432.CCR-06-2300.

53. Kratochwil C, Giesel FL, Bruchertseifer F, Mier W, Apostolidis C, Boll R, et al. (2)(1)(3)Bi-DOTATOC receptor-targeted alpha-radionuclide therapy induces remission in neuroendocrine tumours refractory to beta radiation: a first-in-human experience. Eur J Nucl Med Mol Imaging. 2014;41:2106-19. doi:10.1007/s00259-014-2857-9.

54. Park C, Choi SI, Kim H, Yoo HS, Lee YB. Distribution of Lipiodol in hepatocellular carcinoma. Liver. 1990;10:72-8. doi:10.1111/j.1600-0676.1990.tb00439.x.

55. Imaeda T, Yamawaki Y, Seki M, Goto H, linuma G, Kanematsu M, et al. Lipiodol retention and massive necrosis after lipiodol-chemoembolization of hepatocellular carcinoma: correlation between computed tomography and histopathology. Cardiovasc Intervent Radiol. 1993;16:209-13. doi:10.1007/BF02602962.

56. Jeong JM, Kim YJ, Lee YS, Ko JI, Son M, Lee DS, et al. Lipiodol solution of a lipophilic agent, (188)Re-TDD, for the treatment of liver cancer. Nucl Med Biol. 2001;28:197-204. doi:10.1016/s09698051(00)00208-0.

57. Ocak M, Toklu T, Demirci E, Selçuk N, Kabasakal L. Post-therapy imaging of 225Ac-DOTATATE treatment in a patient with recurrent neuroendocrine tumor. Eur J Nucl Med Mol Imaging. 2020;47:2711-2. doi:10.1007/s00259-020-04725-x.

58. Robertson AKH, Ramogida CF, Rodriguez-Rodriguez C, Blinder S, Kunz P, Sossi V, et al. Multi-isotope SPECT imaging of the (225)Ac decay chain: feasibility studies. Phys Med Biol. 2017;62:4406-20. doi:10.1088/1361-6560/aa6a99.

\section{Tables}


Table 1

Ex vivo biodistribution of [225Ac]Ac-DOTA-TDA in a technical rabbit model

\begin{tabular}{|c|c|c|c|c|c|c|}
\hline & \multicolumn{2}{|c|}{$\begin{array}{l}\text { Tumor-bearing } \\
6 \text { days p.i. }\end{array}$} & \multicolumn{2}{|c|}{$\begin{array}{l}\text { Non-tumor bearing } \\
23.5 \text { hours p.i. }\end{array}$} & \multicolumn{2}{|c|}{$\begin{array}{l}\text { Non-Tumor bearing } \\
28 \text { hours p.i. }\end{array}$} \\
\hline & $\% \mathrm{ID} / \mathrm{g}$ & $\mathrm{Bq} / \mathrm{g}$ & $\% \mathrm{ID} / \mathrm{g}$ & $\mathrm{Bq} / \mathrm{g}$ & $\% \mathrm{ID} / \mathrm{g}$ & $\mathrm{Bq} / \mathrm{g}$ \\
\hline Blood & 0.001 & 4.291 & 0.006 & 161.4 & 0.036 & 592.2 \\
\hline Heart & 0.002 & 5.641 & 0.003 & 94.95 & 0.004 & 59.36 \\
\hline Lungs & 0.014 & 46.23 & 0.028 & 781.0 & 0.023 & 382.0 \\
\hline Liver & 0.233 & 753.2 & 0.153 & 4352 & 0.172 & 2796 \\
\hline Spleen & 0.092 & 296.4 & 0.078 & 2214 & 0.092 & 1495 \\
\hline Kidneys & 0.020 & 64.59 & 0.039 & 1114 & 0.045 & 731.0 \\
\hline Stomach & 0.010 & 30.65 & 0.047 & 1346 & 0.044 & 708.8 \\
\hline Intestines & 0.020 & 826.3 & 0.031 & 880.3 & 0.028 & 449.9 \\
\hline Marrow & 0.018 & 66.02 & 0.013 & 372.1 & 0.013 & 210.1 \\
\hline Whole Bone & \multicolumn{2}{|c|}{ Indistinguishable from background } & 0.054 & 1521 & 0.011 & 182.7 \\
\hline Muscle & 0.001 & 2.637 & 0.001 & 18.54 & 0.002 & 26.93 \\
\hline Gall Bladder & 0.081 & 260.134 & 0.098 & 1600.62 & 0.105 & 2986.55 \\
\hline Bile & 0.023 & 74.533 & 0.091 & 1483.13 & 0.064 & 1812.35 \\
\hline Tumor & 40.62 & 148370 & -- & & - & \\
\hline
\end{tabular}

Figures 
A Actinium-225

B

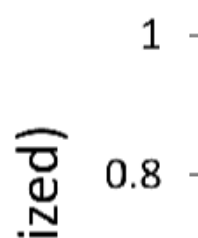

Francium-

221

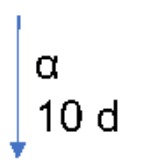

$\alpha$
$4.8 \mathrm{~m}$

Astatine-217

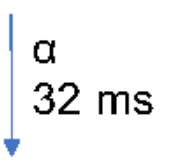

Bismuth-211

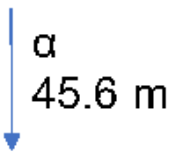

8
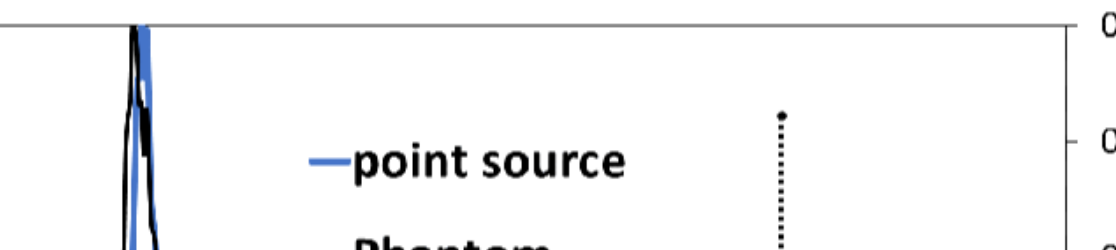

0.3

$\frac{\sqrt{0}}{\mathbb{E}}$

튼 0.6

.6
.4

0.4

틀

0.2

-point source

0.25

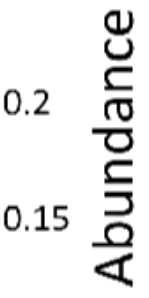

$0.1 \frac{v}{\pi}$

-Phantom

กำ
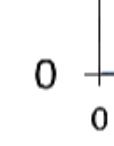

Energy (keV)

Lead-209

Figure 1

(A) Actinium-225 decay scheme [simplified] (B) Energy spectrum of Actinium-225 from a Siemens' SPECT system). Dashed lines indicate gamma and $x$-ray emissions and their abundance, including $218 \mathrm{keV}$ representing Francium-221 and 440 keV representing Bismuth-213 for SPECT imaging.

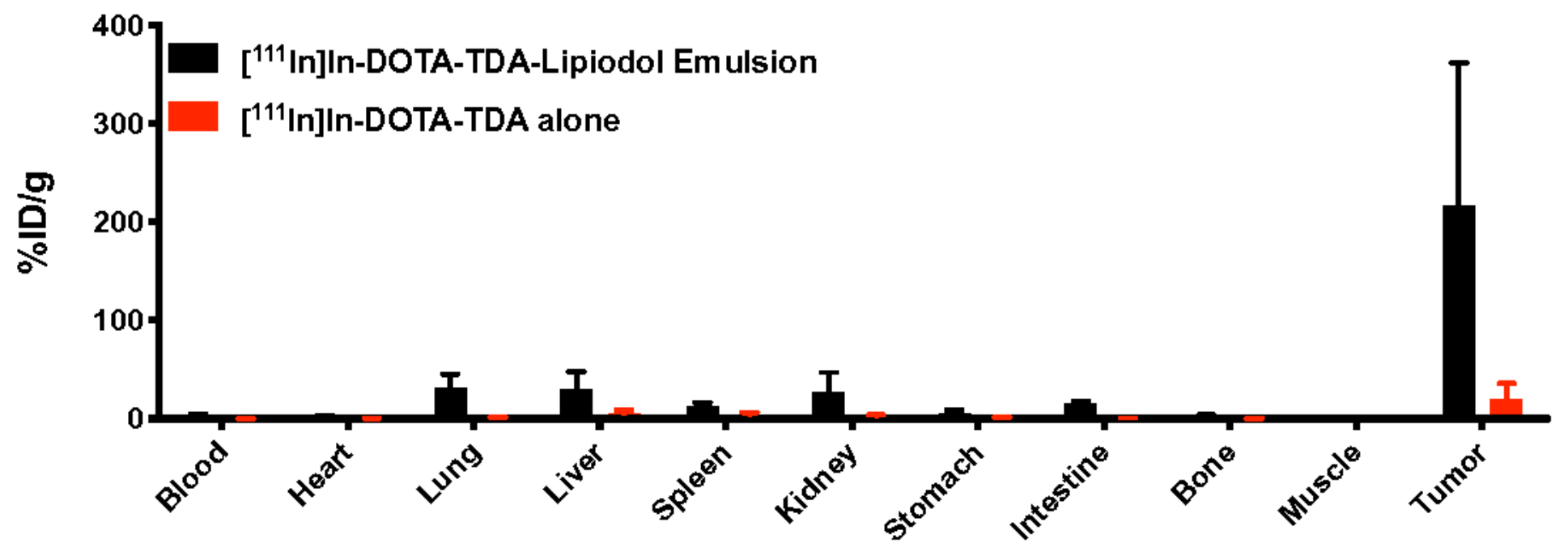

Figure 2

Biodistribution of $111 \mathrm{ln}$-DOTA-TDA $(37 \mathrm{kBq})$ with and without Lipiodol® emulsification in Hep2G tumorbearing NCG mice at 48 hours post-injection $(n=3, p \leq 0.0001)$. 


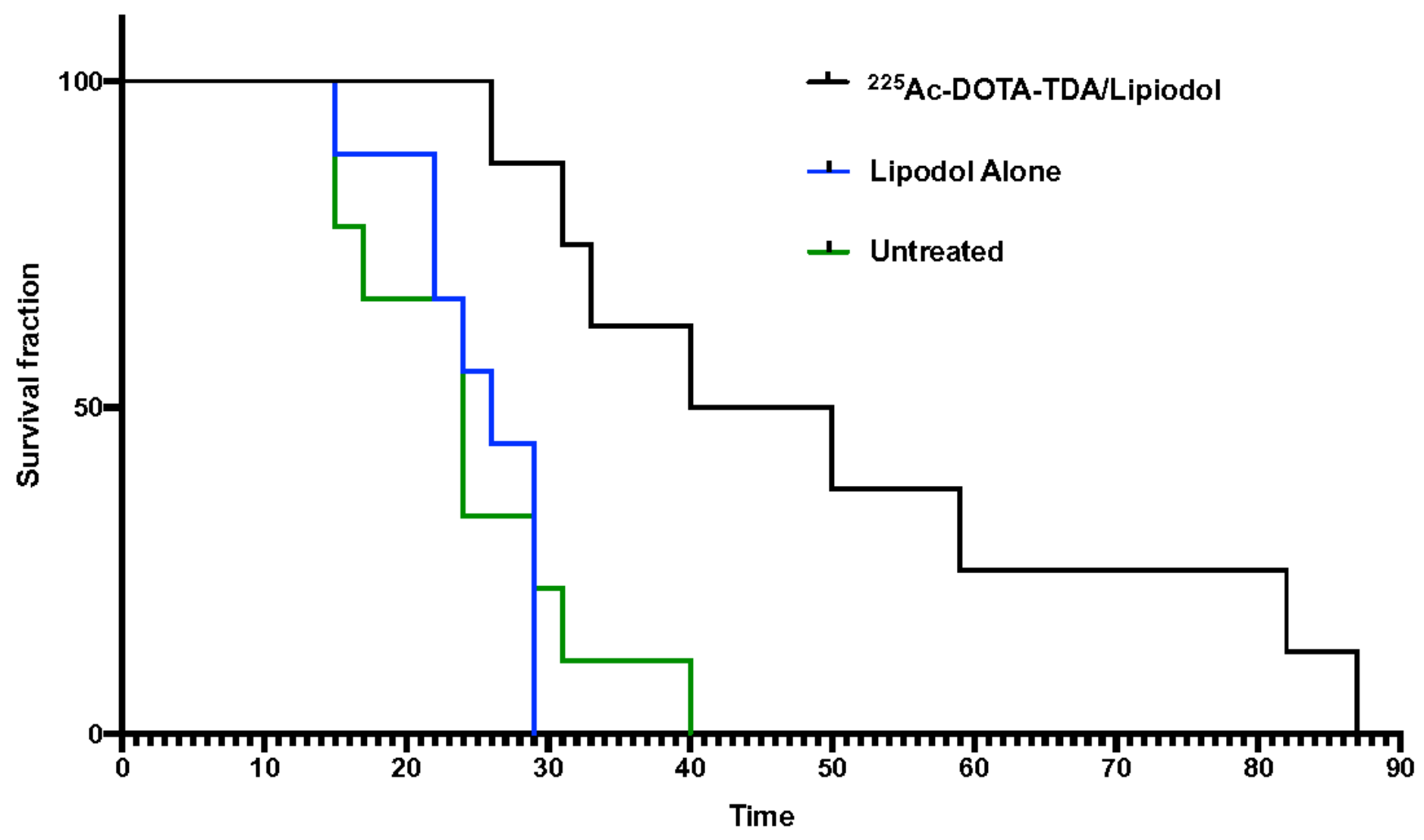

Figure 3

Kaplan-Meier survival plots in Hep2G-tumor bearing mice receiving the following treatments: 1. Saline (60 $\mu \mathrm{L})$ 2. Lipiodol $\circledast$ alone $(60 \mu \mathrm{L})$ or 3. 225Ac-DOTA-TDA/Lipiodol ${ }^{\circledR}(37 \mathrm{kBq}, 60 \mu \mathrm{L})$. 

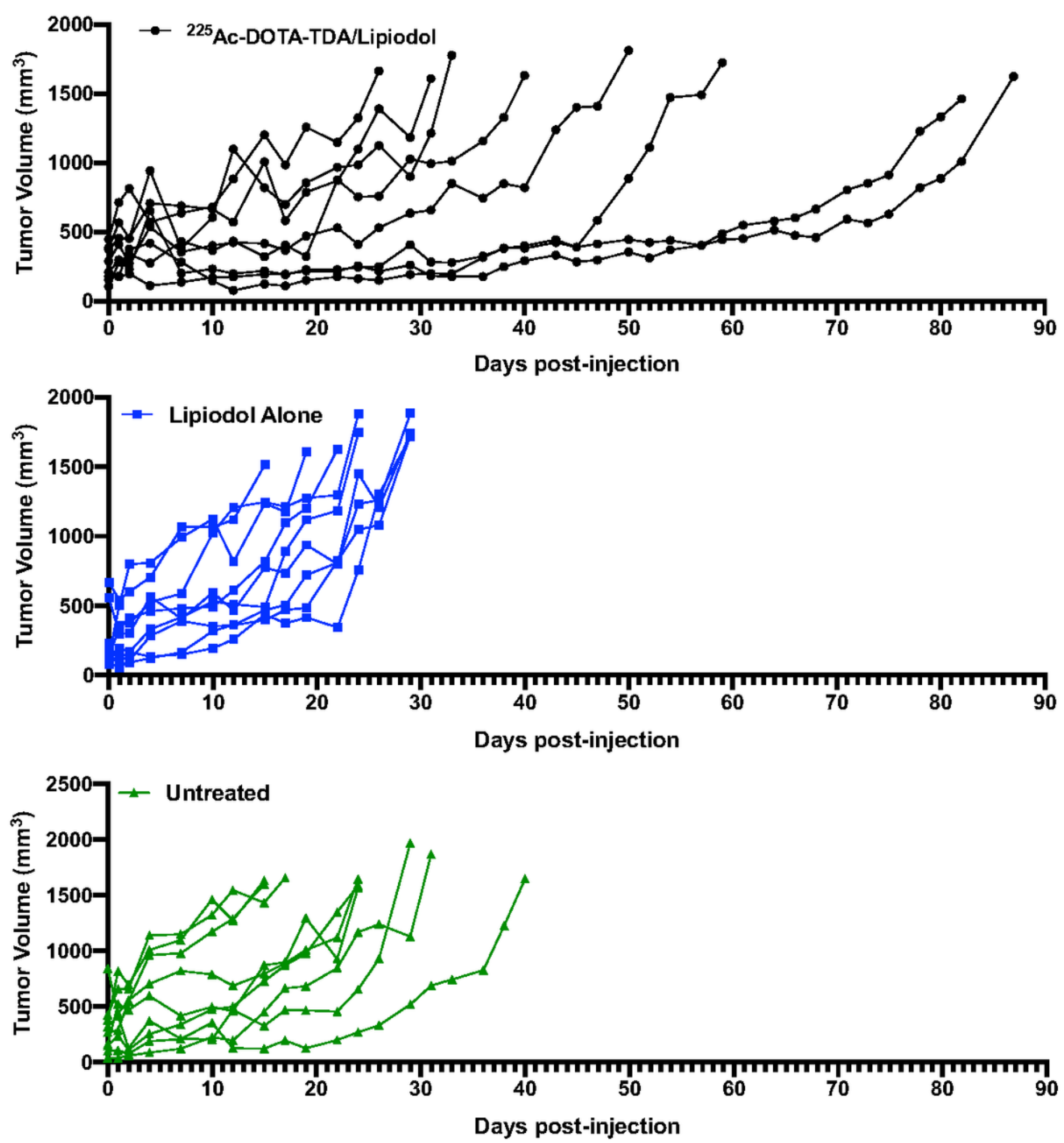

Figure 4

Individual tumor growth curves in a subcutaneous model of liver cancer. NCG mice were injected in the right flank with HEP2G cells when the tumors reached an average volume of $266.5 \pm 23.2 \mathrm{~mm} 3$ the following treatments were administered intratumorally: 1 . Saline $(60 \mu \mathrm{L}) 2$. Lipiodol alone $(60 \mu \mathrm{L})$ or 3 . [225Ac]Ac-DOTA-TDA/Lipiodol (37 kBq, $60 \mu \mathrm{L})$. 
Francium-221

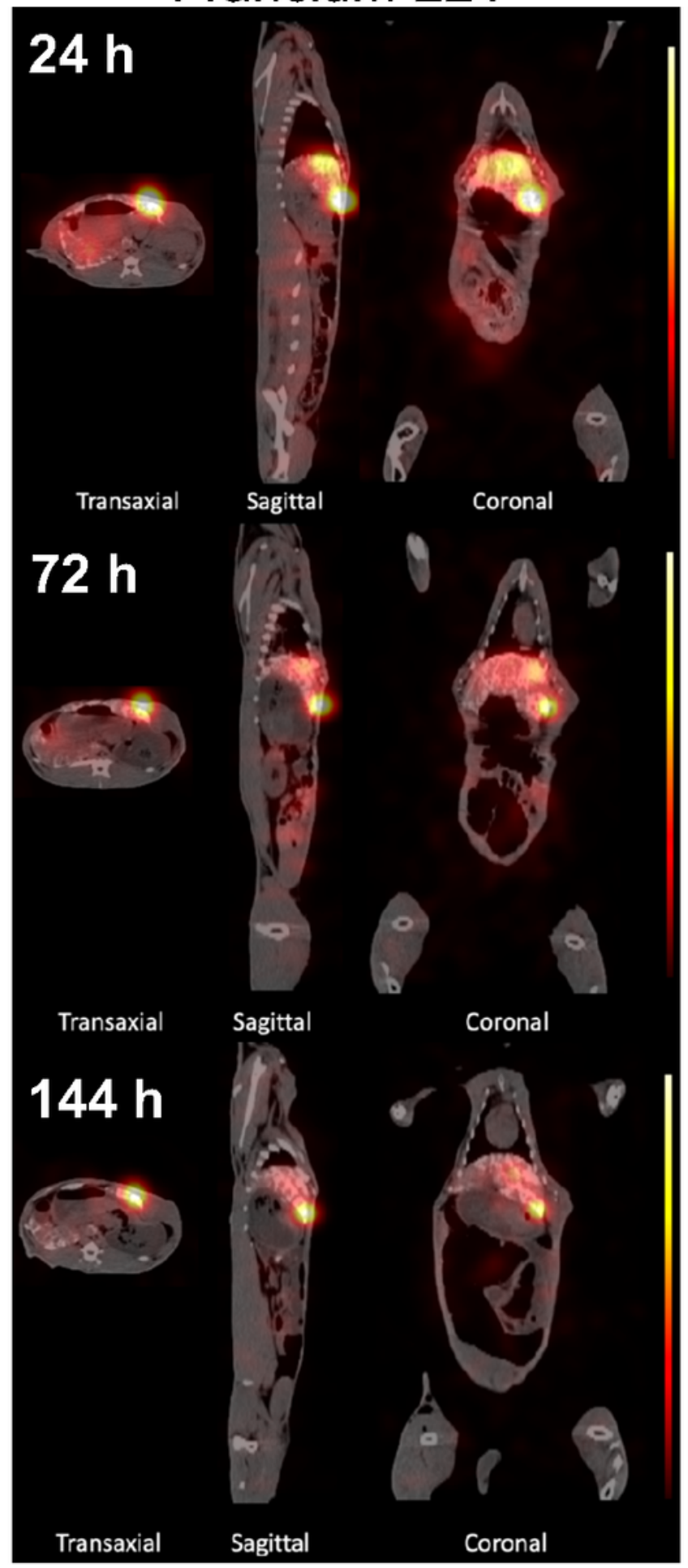

Bismuth-213

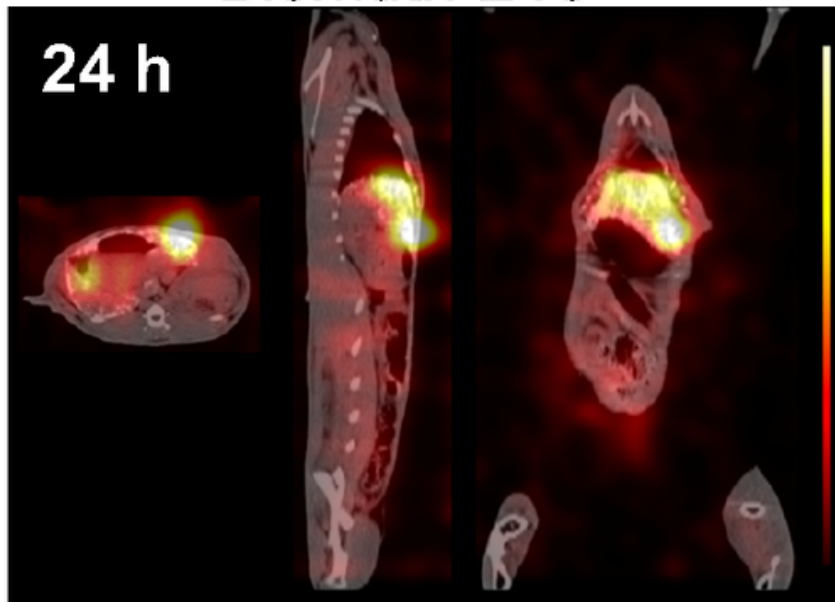

Coronal

Sagittal

$72 \mathrm{~h}$
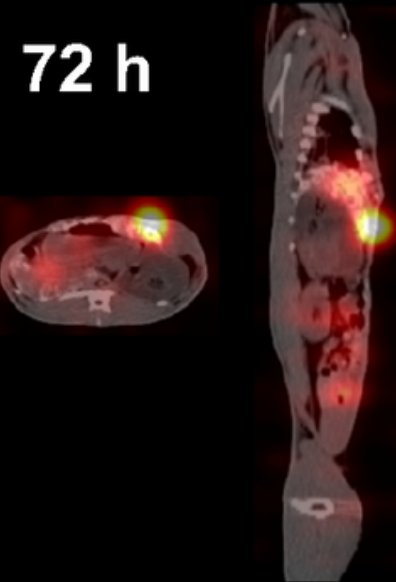

Sagittal

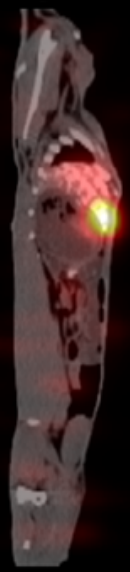

Sagittal

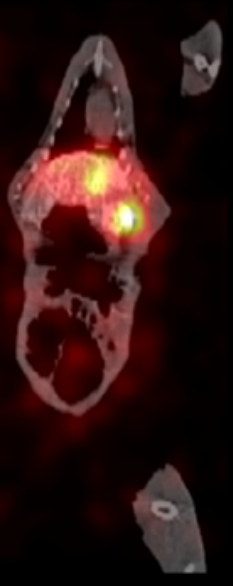

Coronal

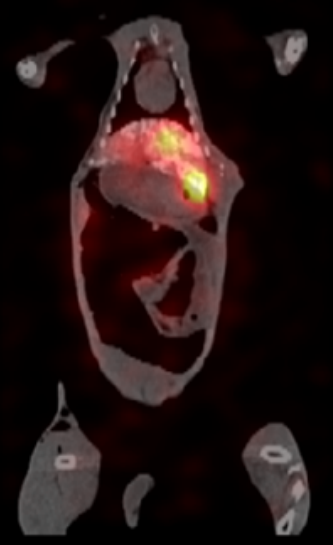

Coronal

\section{Figure 5}

Fused SPECT/CT images of rabbit with a VX2 hepatic tumor at 24, 72 and 144-hours post-injection of [225Ac]Ac-DOTA-TDA/Lipiodol® emulsion. Left Panels: Francium-221 energy window. Right panels: Bismuth-213 energy window. 